\title{
Development of a real-time RT-PCR assay for the detection of pan-human parechoviruses
}

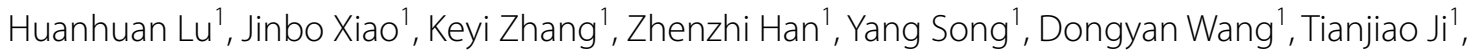 \\ Dongmei Yan ${ }^{1}$, Shuangli Zhu', Wenbo $\mathrm{Xu}^{1,2}$ and Yong Zhang ${ }^{1,2^{*}}$ (])
}

\begin{abstract}
Background: Parechoviruses (PeV-As), which constitute a new genus within the family Picornaviridae, have been associated with numerous localized outbreaks of serious diseases, such as coryza, pneumonia, maculopapular exanthem, and conjunctivitis. However, to the best of our knowledge, only a few laboratories worldwide conduct tests for the identification of this group of viruses. Therefore, in this study, we aimed to develop and validate a real-time RT-PCR assay for the identification of PeV-As.

Methods: To design and validate a real-time PCR primer-probe targeting the 5'-UTR region of PeV-As, the 5'-UTR sequences of PeV-As available in GenBank were aligned using the MUSCLE algorithm in MEGA v7.0. Thereafter, the highly conserved 5'-UTR region was selected, and its primer-probe sequence was designed using Primer Premier v5.0. This primer-probe sequence was then evaluated for specificity, sensitivity, and repeatability, and for its validation, it was tested using fecal samples from 728 healthy children living in Beijing (China).

Results: The PeV-A real-time RT-PCR assay detected only the RNA-positive standards of PeV-A genotypes (1-8, 14, 17, and 18), whereas 72 serotypes of non-PeV-A EV viruses were undetected. In addition, the VP1 region of these 11 PeV-A genotypes that tested positive were amplified using the primers designed in this study. Typing results indicated that eight, one, and two strains of the 11 were PeV-A1, PeV-A4, and PeV-A6, respectively. We also determined and presented the genetic characterization and phylogenetic analyses results corresponding to these 11 VP1 region sequences. Furthermore, real-time RT-PCR assay showed good sensitivity with $L O D$ of $10^{2}$ copies/ $\mu L$. Positive results in eight parallel experiments at each concentration gradient from $10^{7}$ copies $/ \mu \mathrm{L}$ to $10^{2}$ copies $/ \mu \mathrm{L}$, indicating good repeatability.
\end{abstract}

Conclusion: Our findings suggested that the real-time RT-PCR assay developed in this study can be applied for routine PeV-A identification. We detected PeV-A1, 4 and 6 genotypes in the 728 faecal samples using this method. Additionally, we believe that our results will serve as a foundation for further studies on PeV-As and facilitate the expansion of the gene sequence information available in GenBank.

Keywords: Human parechoviruses, VP1 region, Real-time RT-PCR, Typing, Phylogenetics

${ }^{*}$ Correspondence: yongzhang75@sina.com

${ }^{1}$ WHO WPRO Regional Polio Reference Laboratory and National Health

Commission Key Laboratory of Biosafety, National Health Commission

Key Laboratory of Medical Virology, National Institute for Viral

Disease Control and Prevention, Chinese Center for Disease Control and Prevention, Beijing, People's Republic of China

Full list of author information is available at the end of the article

\section{Background}

Human parechoviruses (PeV-As), which are RNA viruses that are closely related to enteroviruses of the family Picornaviridae, were first isolated several decades ago [1]. Of the 19 parechovirus genotypes (PeV-A1-19) that are currently known to infect humans, genotypes 1 and 3 are most commonly associated with diseases [2,3]. It has also been observed that PeV-As, which commonly cause 
high persistent fevers in infants aged less than 5 months, are associated with sepsis-like conditions. Reportedly, the outbreak of a sepsis-like disease in children that occurred in Australia between 2013 and 2014 was caused by PeVA3 [4]. It is also known that maculopapular exanthema, which is a common and distinctive rash involving the extremities, with palmar and plantar erythema is associated with PeV-A3 infection (Table 1). To effectively treat such PeV-A-associated diseases, the development of a sensitive and reproducible PeV-A identification method with good specificity is necessary.

PeV-As are positive-strand RNA viruses belonging to the genus Parechovirus, which is part of the expanding Picornaviridae family. The PeV-A genome is approximately $7300 \mathrm{bp}$ long and divided into four distinct regions, namely, the $5^{\prime}$-untranslated region $\left(5^{\prime}-U T R\right)$, an open reading frame region $(O R F)$, the $3^{\prime}-U T R$ region, and the poly(A) tail region, which is similar to the enterovirus genome [5, 6]. Additionally, PeV-A capsids each comprise 60 copies of three viral proteins, VPO, VP1, and VP3 [7], that cleave post-translationally from a single polyprotein that forms the basic building block of the capsids. Moreover, in most small RNA viruses, VPO is cleaved into fragments $V P 2$ and $V P 4$ following viral particle assembly [8]. Nonetheless, unlike the VP0 of other small-RNA viruses, the $V P O$ of PeV-As remains intact, resulting in this capsid containing only three instead of four distinct polypeptides $[7,9]$. Specifically, human parechoviruses type 1 and 2 (PeV-A1 and PeV-A2, respectively), which were first identified in 1956 during a summer outbreak of diarrhea in children, were classified as echoviruses type 22 and 23 in the genus Enterovirus (EV), respectively [10, 11]. The roles played by these pathogens in human diseases were recently elucidated via molecular diagnostic techniques. In 1997, advances in molecular biology led to the discovery of significant differences between the genomic structures, encoded proteins, and biological properties of these pathogens and those of enteroviruses, resulting in them being renamed parechoviruses type 1 and 2, respectively [12]. The genome length of the structural protein-coding region of $\mathrm{PeV}-\mathrm{A}$ is shorter than that of EV, and the genome length of the non-structural protein-coding region is longer than that of EV. Some of the PeV-A types (PeV-A1-A2, A4-A6) contain arginineglycine-glutamate amino acids (RGD) at the carboxyl terminus of VP1. Among the genus Parechovirus, only $\mathrm{PeV}-\mathrm{A}$ can infect human. Although the 5'-UTR region is conserved in PeV-A, it is distinct from other viruses of picornaviridae, therefore, primers with 5'-UTR region can specifically detect PeV-A. Moreover, similar to enteroviruses, $\mathrm{PeV}$-As, which primarily replicate in the gut, are typically transmitted via the fecal-oral route but can also be transmitted via the nasopharyngeal route [13]. The slow, laborious, and insensitive cell culturing processes that are used to diagnose $\mathrm{PeV}-\mathrm{A}$ infections have been largely replaced by nucleic acid amplification procedures such as reverse transcription polymerase chain reaction (RT-PCR), which can be used to target the conserved 5'-UTR region [5-9]. Furthermore, in 1999, a molecular typing method based on aligning the RT-PCR-amplified sequences of the $V P 1$ coding region with sequence data corresponding to all PeV-A genotypes (the nucleotide sequence consistency in the VP1 region of identical strains was greater than $75 \%$ ) was introduced as a more effective technique for genotype determination $[14,15]$. In summary, for the detection of PeV-A, real-time RTPCR with 5'-UTR region probe-primer is usually used to determine the negative or positive of the samples, and RT-PCR with VP1 region primers is used to further determine the genotype of the positive sample, so as to better understand the epidemiological characteristics of PeV-A.

Therefore, in this study, we developed and validated a real-time RT-PCR assay for the identification of PeVAs using 728 clinical samples. Positive samples were amplified via nested RT-PCR, and the VP1 region was

Table 1 Classification and clinical symptoms of PeV-A

\begin{tabular}{|c|c|}
\hline Genotype of PeV-A & Clinical symptoms \\
\hline PeV-A1 & $\begin{array}{l}\text { Mainly gastrointestinal tract symptoms (diarrhea and emesis) and respiratory tract symptoms (cough, wheezing, rhinorrhea, } \\
\text { tachypnea and herpangina), followed by fever, rash, meningitis, encephalitis and acute flaccid paralysis }\end{array}$ \\
\hline PeV-A3 & $\begin{array}{l}\text { Mainly neonatal fever, meningitis, encephalitis and neonatal sepsis-like disease, followed by gastrointestinal tract symptoms } \\
\text { (diarrhea and emesis), respiratory tract symptoms (cough, wheezing, rhinorrhea, tachypnea and herpangina), rash and acute } \\
\text { flaccid paralysis }\end{array}$ \\
\hline PeV-A4 & $\begin{array}{l}\text { Mainly gastrointestinal tract symptoms (diarrhea and emesis) and respiratory tract symptoms (cough, wheezing, rhinorrhea, } \\
\text { tachypnea and herpangina), followed by Neonatal fever, meningitis and encephalitis }\end{array}$ \\
\hline PeV-A6 & $\begin{array}{l}\text { Gastrointestinal tract symptoms (diarrhea and emesis), respiratory tract symptoms (cough, wheezing, rhinorrhea, tachypnea and } \\
\text { herpangina) }\end{array}$ \\
\hline PeV-A2, 5, 8, 10 & Gastrointestinal tract symptoms (diarrhea and emesis) \\
\hline PeV-A7, 9, 11-19 & Unknown \\
\hline
\end{tabular}


sequenced using the primers designed in this study. We also performed characterization and phylogenetic analysis on the VP1 sequence. The epidemiological characteristics of PeV-A in China were further analyzed through genotyping of the positive samples. The results of this study provide a firm theoretical basis for further studies on PeV-As and help to expand the gene sequence information available in GenBank.

\section{Methods}

\section{Real-time RT-PCR}

\section{Primer and probe design}

The 5'-UTR sequences of PeV-As available in GenBank were aligned using the MUSCLE algorithm in MEGA v7.0 [16]. Based on the results of this multiple sequence alignment, the highly conserved 5 '-UTR region was selected, and the $5^{\prime}-U T R$ primer-probe sequence (Table 2) was designed in accordance with the principles for designing TaqMan RT-PCR primer and probe sequences using Primer Premier v5.0 (Premier Biosoft, Palo Alto, CA, USA). In addition, the designed primer and probe sequences were screened via BLAST to detect the possibility of cross-reactivity with non-PeV-As.

\section{Preparation of positive standards}

Only 11 PeV-A genotypes (1-8, 14, 17, and 18) with 5'$U T R$ region sequences were available in the GenBank database. Thus, the $5^{\prime}-U T R$ regions of these strains, PeVA1 (Harris strain), PeV-A2 (Williamson strain), PeV-A3 (A308/99 strain), PeV-A4 (K251176-02 strain), PeV-A5 (CT86-6760 strain), PeV-A6 (NII561-2000 strain), PeVA7 (PAK5045 strain), PeV-A8 (BR/217/2006 strain), PeV-A14 (V3C strain), PeV-A17 (M36/CI/2014 strain), and PeV-A18 (GhanaA36 886 strain), were used as target sequences. After their synthesis, they were cloned into the p-GEM-T Easy Vector system (Promega, Madison, WI, USA). Thereafter, the plasmids containing these target sequences were cloned by TsingKe Biological
Technology Limited (Beijing, China). Additionally, the plasmids were linearized using restriction endonuclease Sac I, and DNA purification was performed using AMPure XP (Beckman Coulter, CA, USA). The purified linear plasmids were transcribed in vitro using a RiboMAX $^{\mathrm{TM}}$ Large Scale RNA Production System-T7 kit (Promega, Hilden, Germany) and thereafter purified using the RNeasy Mini Kit (Promega, Hilden, Germany). To calculate the copy numbers, the purified RNA was then spectrophometrically quantified using the Qubit ${ }^{\mathrm{TM}}$ RNA BR Assay Kit (Invitrogen, Eugene, OR, USA).

\section{Reagents, reaction systems, and real-time RT-PCR settings}

As a template, $3-\mu \mathrm{L}$ samples of the nucleic acids were used (the total reaction volume was $25 \mu \mathrm{L}$ ) in a One Step PrimeScript $^{\mathrm{TM}}$ RT-PCR Kit (Perfect Real Time, TaKaRa, Dalian, China). The concentrations of each primer and probe in the reaction mixtures were 0.8 and $0.4 \mu \mathrm{M}$, respectively. The reaction settings were as follows: the system was maintained at $42{ }^{\circ} \mathrm{C}$ for $10 \mathrm{~min}$ and $95^{\circ} \mathrm{C}$ for $1 \mathrm{~min}$. This was followed by 40 cycles of amplification at $95{ }^{\circ} \mathrm{C}$ for $10 \mathrm{~s}$ and $55{ }^{\circ} \mathrm{C}$ for $40 \mathrm{~s}$. All the real-time RTPCR experiments were performed using QuantStudio 5 instruments (Applied Biosystems, Thermo Fisher Scientific, Waltham, MA, USA).

\section{Specificity testing}

To assess the specificity of the PeV-A assay, 72 known non-PeV-A strains, including 16 serotypes of EV-A (CVA2-A8, CVA10, CVA12, CVA14, CVA16, EV-A71, EV-A76, EV-A89, EV-A90, and EV-A120), 45 serotypes of EV-B (CVB1-B6, CVA9, E1-7, E9, E11-21, E24-27, E29-33, EV-B74-B75, EV-B79-B81, EV-B83, EV-B85, EV-B93, EV-B97, and EV-B106), and 11 serotypes of EV-C (PV-1, PV-3, CVA1, CVA11, CVA13, CVA17, CVA20-A21, CVA24, EV-C96, and EV-C99) were tested together using 11 types of PeV-A (1-8, 14, 17, and 18) RNA-positive standards. Enterovirus-specific real-time

Table 2 Forward $(F)$ and reverse $(R)$ primers and probe $(P)$ for the detection of human parechovirus (PeV-A) and primers for amplification of the VP1 region of PeV-A

\begin{tabular}{llll}
\hline Name & Primer sequence $\mathbf{( 5}^{\prime} \mathbf{- 3} \mathbf{3}^{\prime} \mathbf{)}$ & $\begin{array}{l}\text { Nucleotide } \\
\text { Position (nt)* }\end{array}$ & References \\
\hline PeV-A-5'UTR-F & TGACGTGTGGGACTTRTTGG & $352-372$ & This study \\
PeV-A-5'UTR-R & CCTTCGTGGGCCYTACAACTAGTG & $538-561$ & This study \\
PeV-A-5'UTR-P & CYCTGGGGCCAAAGCCAAGGTTT & $433-456$ & This study \\
PeV-A-VP1-F1 & TTYTCYACTTGGATGMGGAARA & $2159-2180$ & This study \\
PeV-A-VP1-R1 & ATRCTYGACATHARNCCWGC & $3392-3411$ & This study \\
PeV-A-VP1-F2 & CNTGGGGYTCVCARATGGAYYT & $2340-2361$ & This study \\
PeV-A-VP1-R2 & CCATARTGYTTRTARAAVCCYCTRT & $3081-3105$ & This study \\
\hline
\end{tabular}

* Relative to the Harris PeV-A1 strain 
RT-PCR assays showed that all the non-PeV-A strains were positive. Thereafter, they were stored in our laboratory. Viral RNA was extracted using a QIAamp Viral RNA Mini Kit (Qiagen, Hilden, Germany), in accordance with the manufacturer's instructions.

\section{Sensitivity and stability testing}

Globally, the most common PeV-A types are PeV-A1 and PeV-A3. Therefore, the sensitivity and stability of the probes was evaluated based on these two PeVAs. The limit of detection (LOD) of the real-time RTPCR assay was evaluated via a serial tenfold dilution of PeV-A1 $\left(2.1 \times 10^{8}-2.1 \times 10^{1}\right.$ copies $\left./ \mu \mathrm{L}\right)$ and PeV-A3 $\left(1.9 \times 10^{8}-1.9 \times 10^{1}\right.$ copies $\left./ \mu \mathrm{L}\right)$. The results were interpreted as 'positive' for threshold cycle $(\mathrm{Ct})$ values $\leq 35$ and 'negative' for $\mathrm{Ct}>35$. The obtained PeV-A1 and PeVA3 RNA standards were diluted tenfold. In this regard, the approximate proportion of the $\mathrm{Ct}$ value that could be explained by the copy number was determined based on the coefficient of determination $\left(R^{2}\right)$. Thereafter, seven different concentrations $\left(2.1 \times 10^{7}-2.1 \times 10^{1}\right.$ and $1.9 \times 10^{7}-1.9 \times 10^{1}$ copies $/ \mu \mathrm{L}$ for PeV-A1 and PeV-A3, respectively) were selected as templates based on eight parallel replicate experiments.

\section{Clinical samples for validation Sample material}

To further validate this method, experiments were conducted using stool from healthy children in Beijing. This study involved no human experimentation. Within the June-November 2019 period, a total of 728 stool samples were collected from 549 healthy children living in Beijing, China. All the 549 children, who participated in the study, were aged below 5 years. Of these 549 children, $284(51.73 \%)$ were boys, and 265 (48.29\%) were girls. The stool samples were collected from children living in five districts in Beijing (Haidian, $\mathrm{n}=179$; Daxing, $\mathrm{n}=183$; Dongcheng, $\mathrm{n}=117$; Huairou, $\mathrm{n}=122$; and Fengtai, $\mathrm{n}=127$ ). These samples were sent to the National Institute for Viral Disease Control and Prevention, Chinese Center for Disease Control and Prevention (CDC) for the investigation on enterovirus prevalence in healthy population.

\section{Stool sample treatment and nucleic acid extraction}

All the fecal samples were intended for nucleic acid extraction; therefore, they required special handling. The stool samples (approximately $2 \mathrm{~g}$ each) were dissolved in $10 \mathrm{~mL}$ of phosphate-buffered saline containing antibiotics and thereafter treated with $1 \mathrm{~g}$ of glass beads and $1 \mathrm{~mL}$ of chloroform, followed by vigorous shaking for 20 min using a mechanical shaker and centrifugation for $20 \mathrm{~min}$ at $1500 \times g$ in a refrigerated centrifuge. Then, the supernatant was stored at $-80^{\circ} \mathrm{C}$ until further analysis. RNA was extracted from the stool suspension using a QIAamp Viral RNA Mini Kit (Qiagen, Hilden, Germany).

\section{Comparison of different real-time RT-PCR assays for PeV-A detection}

A TaKaRa One Step PrimeScript ${ }^{\mathrm{TM}}$ RT-PCR Kit (Perfect Real Time) was used to detect viral RNA via real-time RT-PCR using the probe/primer sequences of PeV-A designed in this study and the probe/primer sequences of PeV-A reported in a previous study (AN345, AN344, and AN257) [17]. The reaction settings corresponding to the probe/primer sequences designed in this study were as follows: the system was maintained at $42{ }^{\circ} \mathrm{C}$ for $10 \mathrm{~min}$ and at $95^{\circ} \mathrm{C}$ for $1 \mathrm{~min}$, followed by two 40 cycles of amplification at $95{ }^{\circ} \mathrm{C}$ for $10 \mathrm{~s}$ and at $55{ }^{\circ} \mathrm{C}$ for $40 \mathrm{~s}$. The reaction settings corresponding to primer/probe sequences, AN345, AN344, and AN257, for PeV-A detection were set as previously reported [17].

\section{VP1 region amplification and sequencing and molecular typing}

The VP1 region of PeV-As was amplified via nested PCR using the Primescript One Step RT-PCR Kit Ver.2 (TaKaRa). The first reaction system $(25 \mu \mathrm{L})$ contained $3 \mu \mathrm{L}$ of the original sample template RNA, $1 \mu \mathrm{L}$ of PrimeScript 1 Step Enzyme Mix, $12.5 \mu \mathrm{L}$ of $2 \times 1$ Step Buffer (Dye Plus), $7.5 \mu \mathrm{L}$ of RNase Free $\mathrm{dH}_{2} \mathrm{O}, 0.5 \mu \mathrm{L}$ of forward primer-F1, and $0.5 \mu \mathrm{L}$ of reverse primer-R1 (all the final concentrations were $20 \mu \mathrm{M}$ ). The reaction settings were as follows: the system was maintained at $50{ }^{\circ} \mathrm{C}$ for $30 \mathrm{~min}$ and at $94{ }^{\circ} \mathrm{C}$ for $2 \mathrm{~min}$, followed by 40 cycles of amplification at $94{ }^{\circ} \mathrm{C}$ for $30 \mathrm{~s}, 50{ }^{\circ} \mathrm{C}$ for $30 \mathrm{~s}, 72{ }^{\circ} \mathrm{C}$ for $1 \mathrm{~min}$ $20 \mathrm{~s}$, and finally $72{ }^{\circ} \mathrm{C}$ for $7 \mathrm{~min}$. In the second reaction system, the template was the product of the previous reaction, and the primers were F2/R2 (Table 2). The reaction settings were as follows: the system was maintained at $94{ }^{\circ} \mathrm{C}$ for $2 \mathrm{~min}$, followed by 40 cycles of amplification at $94{ }^{\circ} \mathrm{C}$ for $30 \mathrm{~s}, 50{ }^{\circ} \mathrm{C}$ for $30 \mathrm{~s}, 72{ }^{\circ} \mathrm{C}$ for $1 \mathrm{~min} 20 \mathrm{~s}$, and finally $72{ }^{\circ} \mathrm{C}$ for $7 \mathrm{~min}$. The PCR product was purified using a QIAquick PCR purification kit (Qiagen, Hilden, Germany) and sequenced in both directions using an ABI 3130 Genetic Analyzer (Applied Biosystems, Foster City, CA, USA), resulting in each strand being sequenced at least once. The EV Genotyping Tool and BLAST were used to identify the parechovirus type, based on the obtained partial VP1 sequences [18].

\section{Phylogenetic analysis}

The VP1 sequences of the PeV-A strains were aligned with PeV-A prototype strains using the MUSCLE algorithm implemented via MEGA v7.0 [16]. A nucleotide identity matrix and an amino acid identity matrix were 
generated using BioEdit v7.0.9.0 [19]. Eleven strains as well as the nucleotide and amino acid sequences that showed more than $90 \%$ similarity to those of the VP1 region, based on BLAST, were downloaded to construct a maximum likelihood (ML) tree. The ML trees constructed using the GTR + G model, as suggested by ModelGenerator0.85, were then implemented in MEGA with 1000 bootstrap replicates. In addition, to verify the best topology, ML trees were constructed using RAxML version8.2.12 [20].

\section{Results}

\section{Real-time RT-PCR}

\section{Specificity testing}

In this study, performing the real-time RT-PCR assay for the identification of PeV-As led to the detection of only the RNA-positive standards of PeV-A genotypes (1-8, 14, 17 , and 18), while 72 serotypes of non-PeV-A EV viruses were undetected (Fig. 1).

\section{Sensitivity testing}

To determine the sensitivity of the PeV-A real-time RTPCR assay, serial dilution of RNA transcripts derived from the clones of the $5^{\prime}-U T R$ region of the $11 \mathrm{PeV}-\mathrm{A}$ genotypes $(1-8,14,17$, and 18$)$ was performed, and the corresponding $\mathrm{Ct}$ values were recorded (Fig. 2). Thereafter, standard curves were constructed using the Log value of the RNA copies as the $\mathrm{x}$-axis and the Ct value as the

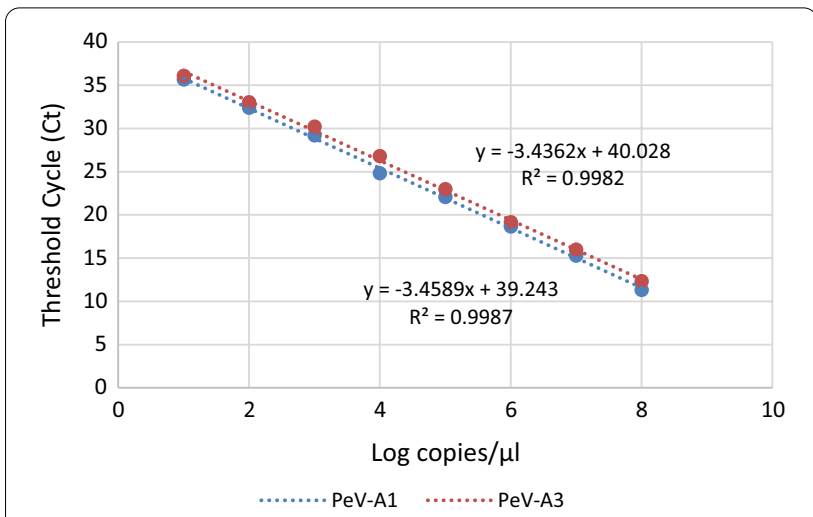

Fig. 2 Dynamic range and linearity of PeV-A real-time assay. Threshold cycle (Ct) values ( $y$-axis) were plotted against tenfold dilutions of PeV-A1 and PeV-A3. $\mathrm{R}^{2}$, regression coefficient

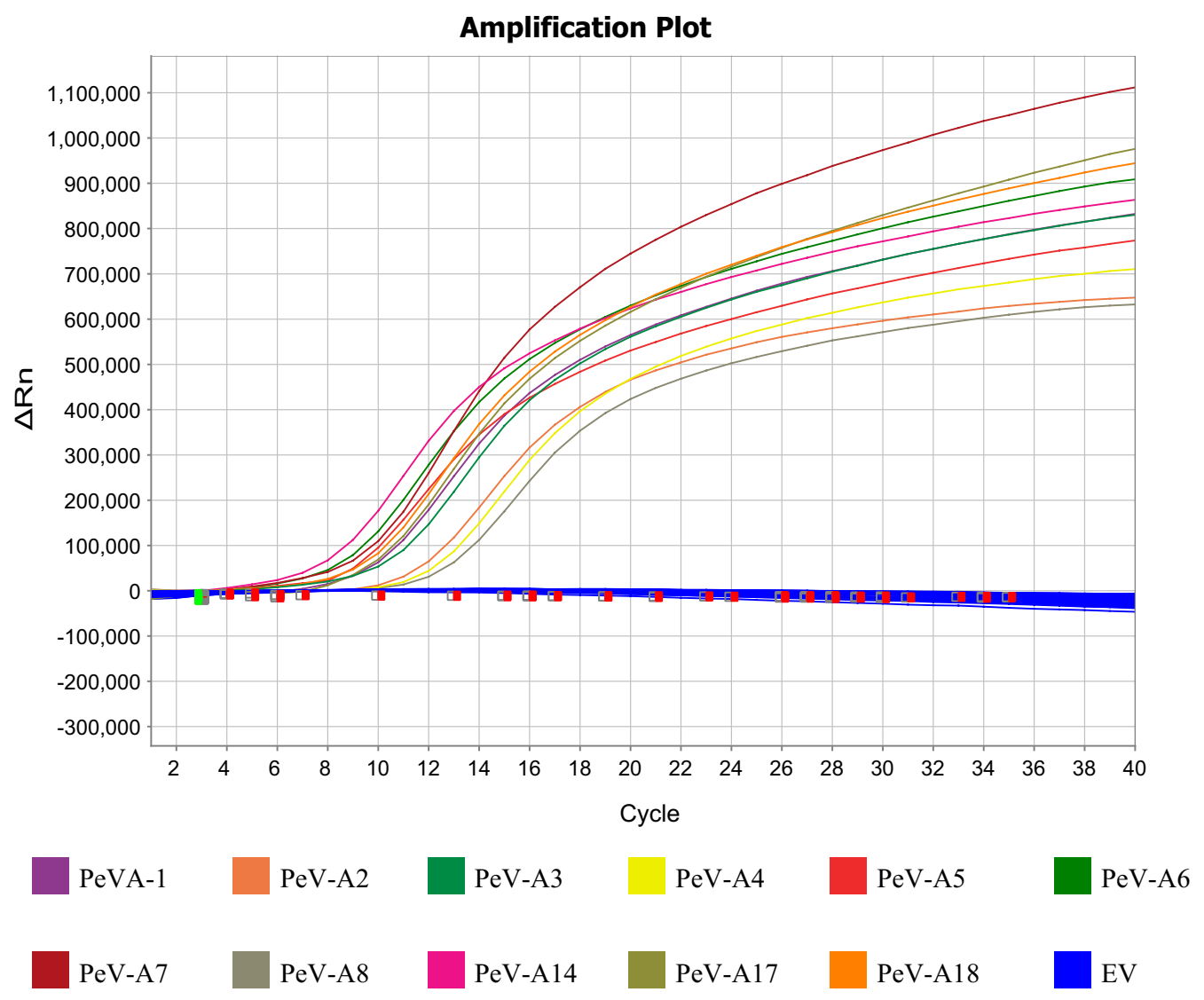

Fig. 1 Evaluation of the specificity of real-time RT-PCR assay for PeV-A detection 
y-axis. The coefficient of determination $\left(R^{2}\right)$ values were 0.9987 and 0.9982 , respectively, and the LODs were both $10^{2}$ copies $/ \mu \mathrm{L}$.

\section{Repeatability testing}

To determine the repeatability of the real-time RT-PCR assay, seven RNA-positive standards with different $\mathrm{PeV}$ $\mathrm{A} 1$ and PeV-A3 concentrations $\left(2.1 \times 10^{7}-2.1 \times 10^{1}\right.$ copies $/ \mu \mathrm{L}$ and $1.9 \times 10^{7}-1.9 \times 10^{1}$ copies $/ \mu \mathrm{L}$, respectively) were tested using eight parallel replicates. Positive results in eight parallel experiments at each concentration gradient from $10^{7}$ copies $/ \mu \mathrm{L}$ to $10^{2}$ copies $/ \mu \mathrm{L}$ (Table 3), indicating good repeatability.

\section{Validation of clinical specimens} Virus detection and genotyping

Using the probe-primer designed in this study, 11 samples tested positive for PeV-A, eight of which were positive with the probe-primers, AN345, AN344, and AN 257. The prevalence of PeV-A infection did not differ significantly between boys and girls $(P>0.1)$. Additionally, the partial VP1 region (696-702 bp) of the 11 strains was successfully determined via RT-PCR, and three types of PeV-As, including PeV-A1 $(\mathrm{n}=8)$, PeV-A4 $(\mathrm{n}=1)$, and

Table 3 The reproducibility of the PeV-A real-time RT- PCR

\begin{tabular}{|c|c|c|}
\hline \multirow[t]{2}{*}{ Copies/reaction } & \multicolumn{2}{|c|}{$\begin{array}{l}\text { No. of positive samples/no. of } \\
\text { samples test by real-time } \\
\text { RT-PCR for detection of PeV-A1 } \\
\text { and PeV-A3 }{ }^{\mathrm{a}}\end{array}$} \\
\hline & PeV-A1 & PeV-A3 \\
\hline $10^{7}$ copies $/ \mu \mathrm{L}$ & $8 / 8$ & $8 / 8$ \\
\hline $10^{6}$ copies $/ \mu \mathrm{L}$ & $8 / 8$ & $8 / 8$ \\
\hline $10^{5}$ copies $/ \mu \mathrm{L}$ & $8 / 8$ & $8 / 8$ \\
\hline $10^{4}$ copies/ $\mu \mathrm{L}$ & $8 / 8$ & $8 / 8$ \\
\hline $10^{3}$ copies/ $\mu \mathrm{L}$ & $8 / 8$ & $8 / 8$ \\
\hline $10^{2}$ copies $/ \mu \mathrm{L}$ & $8 / 8$ & $8 / 8$ \\
\hline $10^{1}$ copies/ $\mu \mathrm{L}$ & $0 / 8$ & $0 / 8$ \\
\hline
\end{tabular}

a The results were interpreted as 'positive' for threshold cycle (Ct) values $\leq 35$ and 'negative' for $\mathrm{Ct}>35$
PeV-A6 $(n=2)$, were identified using the Online Enterovirus Genotyping Tool (Table 4).

\section{Genomic characterization of VP1 region}

The nucleotide sequences of the 11 strains isolated from the stool samples were compared pairwise with those corresponding to the PeV-A1 (Harris strain), PeV-A4 (K251176-02 strain), and PeV-A6 (NII561-2000 strain) prototype strains, as well as other $\mathrm{PeV}-\mathrm{A}$ prototype strains. Similarities between the nucleotide sequences of the $V P 1$ region and the amino acid sequences of eight $\mathrm{PeV}-\mathrm{A} 1$ strains and the PeV-A1 prototype strain were $73.9-75.4 \%$ and $87.9-89.2 \%$, respectively. Additionally, the similarities between the nucleotide sequences of the VP1 region and amino acid sequences of the PeVA4 strain and $\mathrm{PeV}-\mathrm{A} 4$ prototype strain were $82.6 \%$ and $95.6 \%$, respectively, and those between the nucleotide sequences of the $V P 1$ region and amino acid sequences of the two PeV-A6 strains and the PeV-A6 prototype strain were $94.7-95.6 \%$ and $96.9 \%$, respectively.

\section{Phylogenetic analysis of the VP1 region}

A phylogenetic tree was established based on the nucleotide sequences of the VP1 coding regions of the $11 \mathrm{PeV}-\mathrm{A}$ strains identified and all the PeV-A prototype strains in the GenBank database (Fig. 3a). This phylogenetic tree indicated that eight strains clustered with the PeV-A1 prototype strain, while one strain clustered with the PeVA4 prototype strain, and two strains clustered with the PeV-A6 prototype strain, confirming the molecular typing results.

The phylogenetic tree based on the nucleotide sequences downloaded from BLAST and eight VP1 region sequences showed that three of the $\mathrm{PeV}-\mathrm{A} 1$ strains clustered with strains isolated from Belarus, while the remaining five $\mathrm{PeV}-\mathrm{A} 1$ strains clustered with strains isolated from Hong Kong (Fig. 3b). Moreover, the phylogenetic tree based on the PeV-A4 strains showed that the $\mathrm{PeV}-\mathrm{A} 4$ strain clustered with strains isolated from Hong Kong (Fig. 3c), while the two PeV-A6 strains clustered with strains isolated from Hong Kong (Fig. 3d).

Table 4 Comparison of two methods used to test 728 fecal samples

\begin{tabular}{|c|c|c|c|c|}
\hline Virus type & Probe-primer & No. positive & Genotype (no.) ${ }^{b}$ & References \\
\hline \multirow[t]{2}{*}{ PeV-A } & PeV-A-5'UTR-F/R/P & 11 & $\begin{array}{l}\text { PeV-A1 }(n=8) \\
\text { PeV-A4 }(n=1) \\
\text { PeV-A6 }(n=2)\end{array}$ & This study \\
\hline & AN344, AN345, AN257 & 8 & $\begin{array}{l}\text { PeV-A1 }(n=7) \\
\text { PeV-A6 }(n=1)\end{array}$ & [17] \\
\hline
\end{tabular}

${ }^{a}$ Results of real-time RT-PCR test

${ }^{b}$ Result of molecular typing detected via RT-PCR 


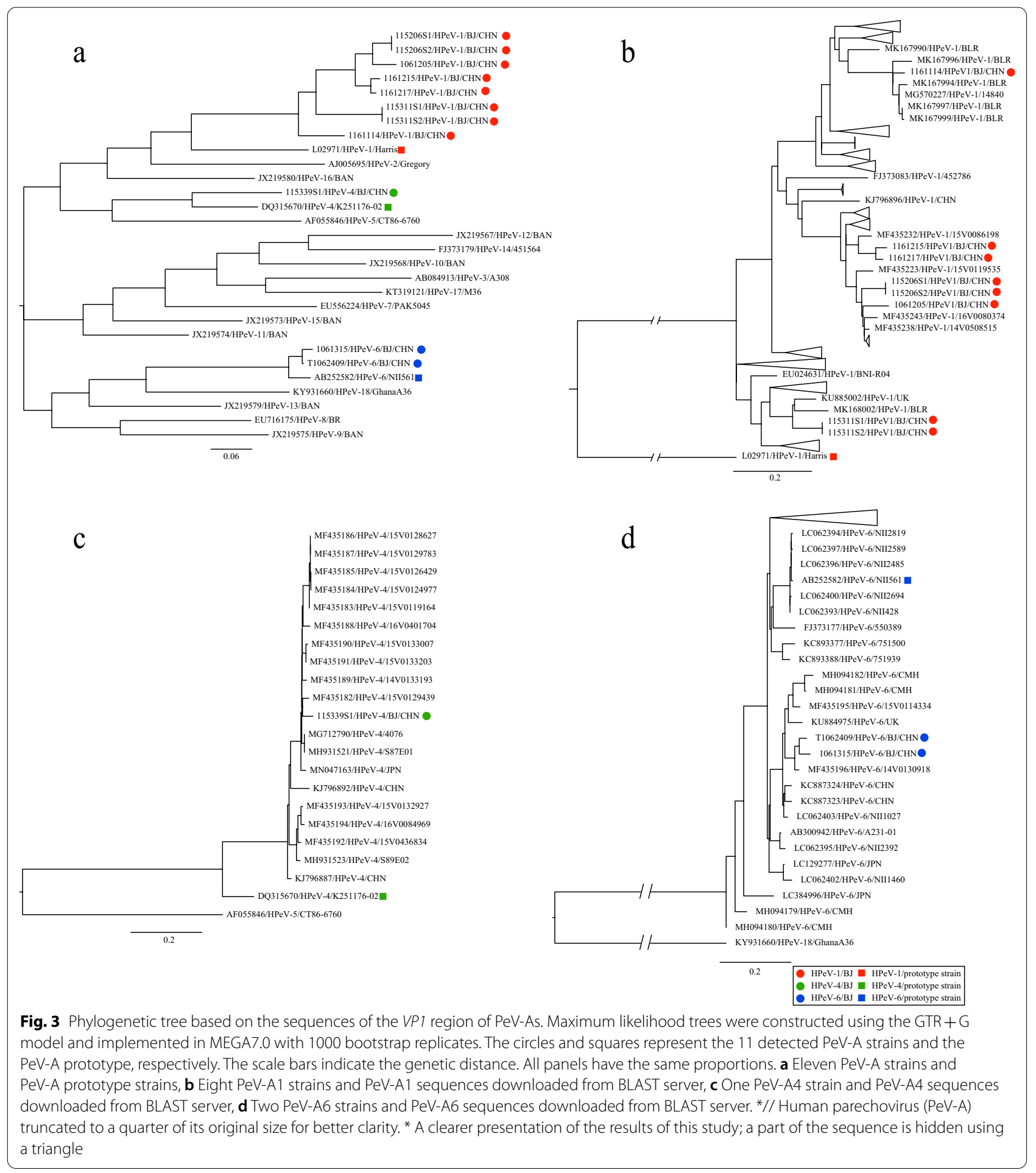

\section{Discussion}

Parechoviruses constitutes a new genus within the Picornaviridae family, and $\mathrm{PeV}-\mathrm{A} 1$, which is considered to be a very common pathogen, is prevalent worldwide [2]. PeV-A3 has caused several global outbreaks of severe diseases in children [3, 4, 21]. Even though parechoviruses and enteroviruses are similar in terms of genomic structure [1], enterovirus testing methods do not detect parechoviruses. Thus, molecular diagnostic methods, such as real-time RT-PCR, are becoming increasingly 
important in disease diagnosis owing to their rapid sensitivity. Furthermore, sensitive nucleotide detection methodologies contribute to a better understanding of the epidemiology and natural history of diseases. Therefore, in this study, we designed and validated a real-time RT-PCR primer-probe targeting the 5'-UTR region of PeV-A. PeV-A-positive standards were tested together with 72 serotypes of known non-PeV-A strains (enteroviruses), and the results showed no cross-reactions and specifically detected only PeV-As. The LOD of this realtime PCR assay was $10^{2}$ copies $/ \mu \mathrm{L}$, indicating that the assay system has good sensitivity. Additionally, positive results in eight parallel experiments at each concentration gradient from $10^{7}$ copies $/ \mu \mathrm{L}$ to $10^{2}$ copies $/ \mu \mathrm{L}$, indicating good repeatability. Given that the 5 '-UTR regions of different types of PeV-As are highly conserved, it is possible that this assay has potential for application in detecting new or emerging PeV-As. The commercial pan-parechovirus techniques, namely: Biomérieux's Biofire (meningo-encephalitis panel) and Fast Track respiratory diagnosis (FTD 33) have their own advantages. Biomérieux's Biofire (meningo-encephalitis panel) has good sensitivity, with 95\% detection limits in CSF of $0.62 \mathrm{TCID}_{50} / \mathrm{mL}$ (PeV-A1) and $9.92 \mathrm{TCID}_{50} / \mathrm{mL}$ (PeV-A) using NucliSENS ${ }^{\circledR}$ easyMAG ${ }^{\circledR}$ and Dx RTS, respectively. It also has good stability: the results of internal variability assay showed that the coefficient of variation ranged between $0.51 \%$ and $0.95 \%$ for PeV-A1 and between $0.66 \%$ and $1.42 \%$ for PeV-A2; The results of the inter variability assay showed that the coefficient of variation ranged between $0.85 \%$ and $2.08 \%$ for HPeV1 and between $1.15 \%$ and $2.90 \%$ for HPeV2. Fast Track respiratory diagnosis (FTD 33) has good sensitivity with the LOD of $10^{3}$ copies/mL and has good stability and specificity. At the same time, they have a slight disadvantage: Biomérieux's Biofire (meningo-encephalitis panel) only verifies PeV-A1 and PeV-A2 in specificity tests, rather than other types of PeV-A; The FTD kit can detect PeV-A, but cannot further determine the genotype of PeV-A. (https://www.biome rieux-diagnostics.com/sites/clinic/files/bes_poster_ ecv2013.pdf) (https://www.siemens-healthineers.com/ molecular-diagnostics/molecular-diagnostics-in-vitrodiagnostics/ftd-respiratory-assays).

When 728 stool samples from healthy children in Beijing, China, were tested using this established real-time PCR method as well as a previous method [17], 11 and eight samples tested positive, respectively. In the process of typing enteroviruses, the 5'-UTR, VP4 and VP2 regions were first chosen for typing, but later it was found that molecular typing based on the VP1 region was more consistent with serotypes, and since enteroviruses are very similar to $\mathrm{PeV}$-A, genotyping of $\mathrm{PeV}$-A based on the $V P 1$ region has been used to date [14, 15]. Nested PCR amplification of the $V P 1$ region was performed on the 11 positive real-time PCR samples, and their VP1 sequences were successfully obtained using the primer sequences designed in this study. Additionally, EV Genotyping Tool, BLAST, and ML tree results, based on the VP1 region, showed that of the 11 strains isolated from the stool samples, eight were $\mathrm{PeV}-\mathrm{A} 1$, one was $\mathrm{PeV}-\mathrm{A} 4$, and two were PeV-A6. Comparisons also revealed that the nucleic acidbased similarity between the eight PeV-A1 strains and the prototype strain was not very high (73.9-75.4\%), and the combination of the results of nucleic acid similarity analysis with the ML tree based on the PeV-A prototype strains indicated that PeV-A1 exhibits a large evolutionary trend in China. In addition, the ML tree, which was established via BLAST analysis, showed that three of the PeV-A1 strains clustered with strains isolated from Belarus, while the remaining five $\mathrm{PeV}-\mathrm{A} 1$ strains, as well as the PeV-A4 and PeV-A6 strains, clustered with strains isolated from Hong Kong. Thus, a majority of the isolates were endemic. Reportedly, parechoviruses are associated with a wide range of gastrointestinal, respiratory, and central nervous system infections [22]. Although parechovirus infections cannot be treated directly, a rapid and sensitive detection method for their identification may reduce hospitalization and shorten the duration of antibiotic treatment. Therefore, we believe that our study lays the foundation for further research on PeV-As and helps expand the gene sequence information available in GenBank.

This study has some limitations. First, although the 5'-UTR region is the most stable region relative to other regions, there is the potential for mutations, deletions and gene recombination over time, further leading to off-target probe-primer and false negative results. Second, due to sample reasons, except for PeV-A1, 4, and 6 genotypes, we did not detect other PeV-A genotypes from the 728 stool samples using this method. In addition, although PeV-A1 and PeV-A3 are the most common types in the world, it is indeed a shortcoming of this study to the determination of LODs for only these two types rather than all standards. Finally, we only performed specificity studies on the enterovirus species A, $\mathrm{B}$, and $\mathrm{C}$ and did not validate the accuracy of our system using other species. Therefore, we think that the realtime RT-PCR method for the detection of pan-PeV-As can be improved in future.

\section{Conclusions}

In this study, we developed and validated a real-time RT-PCR assay for the identification of PeV-As using 728 clinical samples. This real-time RT-PCR assay showed good reproducibility and specificity, and given that the $5^{\prime}-U T R$ regions of different types of PeV-As are 
highly conserved, this assay method possibly has potential for application in the detection of new or emerging PeV-As. In addition, we amplified the VP1 region of the 11 detected PeV-A genotypes using the primers designed in this study. Typing results indicated that eight, one, and two strains were PeV-A1, PeV-A4, and $\mathrm{PeV}-\mathrm{A} 6$, respectively. We also presented the results of the genetic characterization and phylogenetic analyses of $V P 1$ region of these 11 genotype sequences. Therefore, we believe that our study provides a firm foundation for further studies on PeV-As and helps expand the gene sequence information available in GenBank.

\section{Abbreviations}

EV: Enterovirus; 5'-UTR: 5'-Untranslated region; ORF: Open reading frame; 3'-UTR: 3'-Untranslated region; LOD: Limit of detection; Ct: Threshold cycle; SD: Standard deviation; CV: Coefficient of variation; $R^{2}$ : Coefficient of determination; CDC: Chinese Center for Disease Control and Prevention.

\section{Acknowledgements}

We thank the local staff at Beijing Center for Disease Control and Prevention for collecting stool specimens for assay validation.

\section{Authors' contributions}

HL conceived and performed the experiments, analyzed the data, drafted the manuscript, and prepared all the figures. YZ contributed to the conception and design of the experiments, supervised of projects, and polished the manuscript. JX conducted some of the experiments and analyzed the data. $\mathrm{KZ}$ Conducted some of the experiment. ZH, YS, DW, TJ, DY, SZ, and WX analyzed the data. All the authors read and approved the final manuscript.

\section{Funding}

This study was supported by the National key research and development project (Project No. 2021YFC0863000) and the National Key Technology R\&D Program of China (Project Numbers 2018ZX10711001, ZDZX2018ZX10101002-001-003, 2017ZX10104001, and 2018ZX10101002-004-006). We also acknowledge the funding received from Beijing Natural Science Foundation (Project No. L192014). The funding bodies were not involved in the design of the study, clinical sample collection, data analysis, or interpretation. They were also not involved in the writing of the manuscript or in the decision to publish the results.

\section{Availability of data and materials}

VP1 genome nucleotide sequences corresponding to the 11 PeV-A strains determined in this study have been deposited in the GenBank nucleotide sequence database under Accession Numbers MW448141-MW448151.

\section{Declarations}

\section{Ethics approval and content to participate:}

Written informed consent for the use of their clinical samples was obtained from all individuals or their guardians included in the study. The study was approved by the Second Ethics Review Committee of the National Institute for Viral Diseases Control and Prevention (IVDC), Chinese Center for Diseases Control and Prevention, all experimental protocols were approved by IVDC and the methods were carried out in accordance with the approved guidelines.

\section{Consent for publication}

Not applicable.

\section{Competing interests}

The authors declare that they have no competing interests.

\section{Author details}

${ }^{1}$ WHO WPRO Regional Polio Reference Laboratory and National Health Commission Key Laboratory of Biosafety, National Health Commission Key Laboratory of Medical Virology, National Institute for Viral Disease Control and Prevention, Chinese Center for Disease Control and Prevention, Beijing, People's Republic of China. ${ }^{2}$ Center for Biosafety Mega-Science, Chinese Academy of Sciences, Wuhan 430071, People's Republic of China.

Received: 21 July 2021 Accepted: 1 November 2021 Published online: 20 November 2021

References

1. Tang JW, Bendig JW, Ossuetta I. Vertical transmission of human echovirus 11 at the time of Bornholm disease in late pregnancy. Pediatr Infect Dis J. 2005;24(1):88-9.

2. Grist NR, Bell EJ, Assaad FJPMV. Enteroviruses in human disease. Prog Med Virol. 1978;24:114-57.

3. Khatami A, McMullan BJ, Webber M, Stewart P, Francis S, Timmers KJ, et al. Sepsis-like disease in infants due to human parechovirus type 3 during an outbreak in Australia. Clin Infect Dis. 2015;60(2):228-36.

4. Mizuta K, Yamakawa T, Nagasawa H, Itagaki T, Katsushima F, Katsushima Y, et al. Epidemic myalgia associated with human parechovirus type 3 infection among adults occurs during an outbreak among children: findings from Yamagata, Japan, in 2011. J Clin Virol. 2013;58(1):188-93.

5. Arola A, Santti J, Ruuskanen O, Halonen P, Hyypiä T. Identification of enteroviruses in clinical specimens by competitive PCR followed by genetic typing using sequence analysis. J Clin Microbiol. 1996;34(2):313-8.

6. Chapman NM, Tracy S, Gauntt CJ, Fortmueller U. Molecular detection and identification of enteroviruses using enzymatic amplification and nucleic acid hybridization. J Clin Microbiol. 1990;28(5):843-50.

7. Hyypiä T, Auvinen P, Maaronen M. Polymerase chain reaction for human picornaviruses. J Gen Virol. 1989;70(Pt 12):3261-8.

8. Olive DM, Al-Mufti S, Al-Mulla W, Khan MA, Pasca A, Stanway G, et al. Detection and differentiation of picornaviruses in clinical samples following genomic amplification. J Gen Virol. 1990;71(Pt 9):2141-7.

9. Zoll GJ, Melchers WJ, Kopecka H, Jambroes G, van der Poel HJ, Galama JM. General primer-mediated polymerase chain reaction for detection of enteroviruses: application for diagnostic routine and persistent infections. J Clin Microbiol. 1992;30(1):160-5.

10. Hyypiä T, Horsnell C, Maaronen M, Khan M, Kalkkinen N, Auvinen P, et al A distinct picornavirus group identified by sequence analysis. Proc Natl Acad Sci USA. 1992;89(18):8847-51.

11. Wigand R, Sabin AB. Properties of ECHO types 22, 23 and 24 viruses. Arch Gesamte Virusforsch. 1961;11:224-47.

12. Ghazi F, Hughes PJ, Hyypiä T, Stanway G. Molecular analysis of human parechovirus type 2 (formerly echovirus 23). J Gen Virol. 1998;79(Pt 11):2641-50.

13. Esposito S, Rahamat-Langendoen J, Ascolese B, Senatore L, Castellazzi L, Niesters HGM. Pediatric parechovirus infections. J Clin Virol. 2014;60(2):84-9.

14. Oberste MS, Maher K, Kilpatrick DR, Flemister MR, Brown BA, Pallansch MA. Typing of human enteroviruses by partial sequencing of VP1. J Clin Microbiol. 1999:37(5):1288-93.

15. Oberste MS, Maher K, Flemister MR, Marchetti G, Kilpatrick DR, Pallansch MA. Comparison of classic and molecular approaches for the identification of untypeable enteroviruses. J Clin Microbiol. 2000;38(3):1170-4.

16. Kumar S, Stecher G, Tamura K. MEGA7: molecular evolutionary genetics analysis version 7.0 for bigger datasets. Mol Biol Evol. 2016;33(7):1870-4

17. Allan W, Nix, Kaija, Maher, Susanne E, Johansson, et al. Detection of all known parechoviruses by real-time PCR. 2008.

18. Kroneman A, Vennema H, Deforche K, v d Avoort H, Peñaranda S, Oberste MS, et al. An automated genotyping tool for enteroviruses and noroviruses. Journal of clinical virology : the official publication of the Pan American Society for Clinical Virology. 2011;51(2):121-5.

19. Hall TA. BioEdit : a user-friendly biological sequence alignment editor and analysis program for Windows 95/98/NT. Nucl Acids Symp Ser. 1999. 
20. Stamatakis A. RAxML version 8: a tool for phylogenetic analysis and post-analysis of large phylogenies. Bioinformatics (Oxford, England). 2014;30(9):1312-3.

21. Ljubin-Sternak S, Juretić E, Šantak M, Pleša M, Forčić D, Vilibić-Čavlek T, et al. Clinical and molecular characterization of a parechovirus type 1 outbreak in neonates in Croatia. J Med Virol. 2011;83(1):137-41.

22. Ehrnst A, Eriksson M. Epidemiological features of type 22 echovirus infection. Scand J Infect Dis. 1993;25(3):275-81.

\section{Publisher's Note}

Springer Nature remains neutral with regard to jurisdictional claims in published maps and institutional affiliations.
Ready to submit your research? Choose BMC and benefit from:

- fast, convenient online submission

- thorough peer review by experienced researchers in your field

- rapid publication on acceptance

- support for research data, including large and complex data types

- gold Open Access which fosters wider collaboration and increased citations

- maximum visibility for your research: over 100M website views per year

At BMC, research is always in progress.

Learn more biomedcentral.com/submissions 